\title{
Quantum Entanglement of Dark Matter
}

\author{
Jae-Weon Lee* \\ Department of Renewable Rnergy, Jungwon University, 85 Munmu-ro, \\ Goesan-eup, Goesan-gun, Chungcheongbuk-do, 367-805, Korea, and \\ Department of Physics, North Carolina State University, Raleigh, NC 27695, USA
}

\begin{abstract}
We suggest that the dark matter in the universe has quantum entanglement if the dark matter is a Bose-Einstein condensation of ultra-light scalar particles. In this theory, any two regions of a galaxy are quantum entangled due to the quantum nature of the condensate. We calculate the entanglement entropy of a typical galactic halo, which turns out to be at least $O(\ln (\mathrm{M} / \mathrm{m}))$, where $M$ is the mass of the halo and $m$ is the mass of a dark matter particle. The entanglement can be inferred from the rotation curves of the galaxy or the interference patterns of the dark matter density.
\end{abstract}

PACS numbers: 98.62.Gq, 95.35.+d, 03.75.Gg

Keywords: dark matter, BEC, Entanglement, galactic halos

\section{INTRODUCTION}

Dark matter (DM) is one of the greatest mysteries in physics and astronomy. Despite decade-long efforts, the origin of DM defies a successful explanation. The flatness of the galactic rotation curves implies the presence of invisible DM in galactic halos [1], and any plausible DM theory should explain the curves. Cold dark matter $(\mathrm{CDM})$ with the cosmological constant $(\Lambda \mathrm{CDM})$ model ' is very successful in explaining the large-scale structure ' of the universe, but it encounters many difficulties at the galactic scale and below. For example, numerical simulations with the $\Lambda$ CDM model predict a cusped central density and too many subhalos compared to observations [2 [5].

Although several proposals consider dark subhalos or baryonic feedback mechanisms to alleviate these concerns, the consideration of an alternative to CDM playing the role of CDM at the super-galactic scale and at the same time suppressing sub-galactic structures is desirable. Bose-Einstein condensate (BEC) DM or scalar ' field dark matter (SFDM) [6, 7] may be a good alternative to CDM. In this model, DM is a BEC of ultra-light 'scalar particles with mass $m \simeq 10^{-22} \mathrm{eV}$ [8], which implies a large DM Compton wavelength comparable to a galaxy core. This long wavelength prevents the formation of DM-dominated structures smaller than a dwarf galaxy. Beyond this scale, the coherent nature of BEC DM makes it behave like CDM, hence solving the problems of CDM. This behavior was confirmed by a recent high precision numerical study [9, 10].

The idea that DM is an ultra-light boson condensate has a long history. (See Refs. $11-16$ for a review.) Baldeschi et al. 17] considered galactic halos of self-gravitating bosons, and Membrado et al. [18] obtained the rotation curves for a self-gravitating boson sphere. Sin [6] suggested that galactic halos were like gigantic atoms made

\footnotetext{
* scikid@jwu.ac.kr
}

of ultra-light BEC DM such as pseudo Nambu-Goldstone bosons. In that model, the boson DM particles are described by using a single macroscopic wave function of $k p c$ size, and the quantum uncertainty principle prevents halos from self-gravitational collapse. In the context of field theory and general relativity, Lee and Koh [7] suggested that DM halos are giant boson stars described by the Einstein-Klein-Gordon (EKG) Equations. Widrow and Kaiser [19] suggested a new numerical method utilizing the Schrödinger equation for collisionless matter. Similar ideas have been developed by many authors in terms of fuzzy DM, BEC DM, wave DM or ultra-light axions [8, 20 36]. These models of dark mater (Scalar field dark matter: SFDM hereafter) have been shown to be able to explain the observed rotation curves [22, 3741], the large scale structures of the universe [9], the minimum mass and the size of galaxies [42, 43], the cosmic background radiation, spiral arms 44 and more. Works have also been done on the stability of BEC halos [45].

On the other hand, quantum entanglement (a nonlocal quantum correlation) [46] is an important physical resource for quantum information processing such as the quantum key distribution and quantum computing. Furthermore, entanglement is considered as a new order parameter for condensed matter systems such as a BEC. Recently, entanglement was proposed to be the key to understand dark energy [47], gravity [48] and even the spacetime itself [49]. Several other works link cosmology with entanglement. (See Ref. 50 for a review.) These works usually focuse on the generation of quantum field entanglement during the cosmic expansion, especially during inflation [51]. Quantum fluctuation, and hence, quantum entanglement of the cosmic vacuum can influence the density perturbation during and after the inflationary phase. From these perspectives, understanding the entanglement among DM particles is meaningful.

In this paper, we show that the SFDM in the universe has entanglement among DM particles. Although DM makes up about $26 \%$ of the universe, its entanglement has not been studied so far. Because BEC shows a macroscopic quantum behavior by definition, an ex- 
pectation that the SFDM would also have galactic-scale quantum entanglement is reasonable. In section II, we study the entanglement of the SFDM in a dwarf galaxy. In section III, we show how to decide the entanglement from observations. In section IV, we discuss our findings.

\section{ENTANGLEMENT OF BOSE-EINSTEIN CONDENSATE DARK MATTER}

SFDM behaves as a coherent wave with a $k p c$ wavelength while conventional CDM is usually treated as classical point particles. In the SFDM model [6], the galactic DM halo is a single giant boson star, which can be described with a wave function $\psi(\mathbf{r})$ of the non-linear Schrödinger equation (the Gross-Pitaevskii equation ) or equivalently with the following Schrödinger-Poisson equations (SPEs):

$$
\begin{aligned}
i \hbar \partial_{t} \psi & =-\frac{\hbar^{2}}{2 m} \nabla^{2} \psi+m \Phi \psi, \\
\nabla^{2} \Phi & =4 \pi G m|\psi|^{2}
\end{aligned}
$$

with a self-gravitation potential $\Phi$, where $m$ is the mass of an individual DM particle. Note that the number density of local dark matter is proportional to $|\psi(r)|^{2}$. $\Phi$ plays the role of a trap potential for the galactic BEC. For simplicity, we ignore visible matter in this paper, which is a good approximation for DM-dominated galaxies. The SPEs can be obtained from the mean field approximation of a many-body BEC Hamiltonian or the Newtonian approximation of the SFDM Lagrangian [7].

For the study of the entanglement of the SFDM, the SPEs are not enough, and we need a multi-particle formalism. In the many-body theory of a BEC, one usually decomposes the field operator with the annihilation operator $a_{\alpha}$ as

$$
\hat{\Psi}(\mathbf{r})=\sum_{\alpha} \psi_{\alpha}(\mathbf{r}) a_{\alpha},
$$

where the single-particle wave functions $\psi_{\alpha}(\mathbf{r})$ are orthonormal, i.e.,

$$
\sum_{\alpha} \psi_{\alpha}(\mathbf{r}) \psi_{\alpha}^{*}\left(\mathbf{r}^{\prime}\right)=\delta\left(\mathbf{r}-\mathbf{r}^{\prime}\right)
$$

Then, $\hat{\Psi}(\mathbf{r})$ fulfills the commutation relation $\left[\hat{\Psi}(\mathbf{r}), \hat{\Psi}^{\dagger}\left(\mathbf{r}^{\prime}\right)\right]=\delta\left(\mathbf{r}-\mathbf{r}^{\prime}\right) \mathbf{1}$. The SFDM has a high phase-transition temperature $\left(T_{c} \gg T e V\right)$, and the present temperature of DM is so low that we can treat a SFDM state as a zero-temperature BEC state. At zero temperature, all boson particles are in the ground state, which is represented by

$$
\left|\Psi_{0}\right\rangle=\frac{a_{0}^{\dagger N}}{\sqrt{N !}}|0\rangle,
$$

where $N$ is the number of DM particles, $|0\rangle$ denotes the vacuum state, and $a_{0}^{\dagger}=\int d^{3} \mathbf{r} \psi_{0}(\mathbf{r}) \hat{\Psi}^{\dagger}(\mathbf{r}) . \quad \psi_{0}(\mathbf{r})$ is the lowest energy mode function for a single particle in a boson halo, which can be obtained by solving the SPEs (Eq. (1)) .

Studies on the entanglement of a BEC in labs usually focuses on a spinor BEC or a multi-component BEC. However, for our purpose, we need a formalism for a single-component scalar BEC. Following the formalisms in Refs. 52 and 53, we consider the entanglement between two subregions, A and B, of a galaxy. For example, the regions $A$ and $B$ can be the central part and an outer part of a galaxy, respectively.

For the calculation of the entanglement between two regions of a galaxy, annihilation operators $\left(a_{A}, a_{B}\right)$ for subsystems are conveniently defined as

$$
\begin{aligned}
& a_{A}^{\dagger}=\frac{1}{P_{A}} \int_{\mathbf{r} \in A} d^{3} \mathbf{r} \psi_{0}(\mathbf{r}) \hat{\Psi}^{\dagger}(\mathbf{r}), \\
& a_{B}^{\dagger}=\frac{1}{P_{B}} \int_{\mathbf{r} \in B} d^{3} \mathbf{r} \psi_{0}(\mathbf{r}) \hat{\Psi}^{\dagger}(\mathbf{r})
\end{aligned}
$$

where $P_{A}=\int_{\mathbf{r} \in A} d^{3} \mathbf{r}\left|\psi_{0}(\mathbf{r})\right|^{2}=1-P_{B}=1-$ $\int_{\mathbf{r} \in B} d^{3} \mathbf{r}\left|\psi_{0}(\mathbf{r})\right|^{2}$. This means that $a_{A}^{\dagger}$ creates a DM particle in subregion $\mathrm{A}$, and the probability to find the particle there is $P_{A}$. Then, the ground state becomes

$$
\begin{aligned}
\left|\Psi_{0}\right\rangle & =\frac{1}{\sqrt{N !}}\left(\sqrt{P_{A}} a_{A}^{\dagger}+\sqrt{P_{B}} a_{B}^{\dagger}\right)^{N}|0\rangle \\
& =\frac{1}{\sqrt{N !}} \sum_{k=0}^{N}{ }_{N} C_{k} \sqrt{P_{A}^{k} P_{B}^{N-k}} a_{A}^{\dagger k} a_{B}^{\dagger(N-k)}|0\rangle \\
& =\sum_{k=0}^{N} \sqrt{{ }_{N} C_{k} P_{A}^{k} P_{B}^{N-k}}|k\rangle_{A}|N-k\rangle_{B} \\
& \equiv \sum_{k=0}^{N} \sqrt{\lambda_{k}}|k\rangle_{A}|N-k\rangle_{B},
\end{aligned}
$$

where $a^{\dagger k}|0\rangle=\sqrt{k !}|k\rangle$ and $\lambda_{k}={ }_{N} C_{k} P_{A}^{k} P_{B}^{N-k}$. Note that the final state is not separable; thus, any part of DM in a galactic halo is entangled with the DM in other regions in the same galaxy.

The measure of entanglement we choose is the entanglement entropy

$$
S_{E}=-\sum_{k} \lambda_{k} \ln \lambda_{k}
$$

which is the entropy of the reduced density matrix $\rho_{A} \equiv$ $\operatorname{Tr}_{B}\left(\left|\Psi_{0}\right\rangle\left\langle\Psi_{0}\right|\right)$. As $N \rightarrow \infty$, the De Moivre-Laplace theorem

$$
\lambda_{k}={ }_{N} C_{k} P_{A}^{k} P_{B}^{N-k} \simeq \frac{1}{\sqrt{2 \pi N P_{A} P_{B}}} \exp \left(-\frac{\left(k-N P_{A}\right)^{2}}{2 N P_{A} P_{B}}\right)
$$

implies that the reduced density matrix has a normal 
distribution with entropy

$$
\begin{aligned}
S_{E}\left(\lambda_{k}\right) & \simeq \frac{1}{2} \ln \left(2 \pi e N_{A} P_{B}\right) \\
& =\frac{1}{2} \ln \left(2 \pi e \frac{M_{A}}{m}\left(1-\frac{M_{A}}{M}\right)\right) \\
& =\frac{1}{2} \ln \left(\frac{M_{A}}{m}\left(1-\frac{M_{A}}{M}\right)\right)+1.42,
\end{aligned}
$$

where $N_{A}=N P_{A}$ is the mean particle number in subregion $A$ and $M_{A}=m N_{A}$ is the mass of the subregion A. Note that by definition $S_{E}$ is symmetric under an interchange of $A$ and $B$. This means $S_{E}$ has a maximum value $\frac{1}{2} \ln \left(\pi e N_{A}\right)$ when $P_{A}=P_{B}=1 / 2$.

For $m=10^{-22} \mathrm{eV}$ [8] and $M_{A} \ll M$,

$$
S_{E}\left(\lambda_{k}\right) \simeq \frac{1}{2} \ln \left(M_{A} / M_{\odot}\right)+102.42
$$

with the solar mass $M_{\odot}$. For a typical galaxy, the halo mass is $M \simeq 10^{11} M_{\odot}$, so we expect $S_{E}=O\left(10^{2}\right)$. This is not a big number, but this value is just for the entanglement for a specific bisection of a galaxy. Furthermore, entanglement may exist between other modes we did not consider. Therefore, $S_{E}$ in Eq. (10) should be treated as a lower bound for the entanglement of a galaxy.

To see how the entanglement scales, we consider a bipartite system that consists of a spherical subregion (A) within a radial distance $r$ from the center of galaxy and an outer region (B) beyond $r$. These regions could be parts of dwarf galaxies or a solitonic core and a surrounding cloud of BEC, as shown in Ref. 9. We first need to know the wave function to study the entanglement.

The DM mass enclosed inside $r$ is given by

$$
M_{A}(r)=4 \pi m \int_{0}^{r} d r^{\prime} r^{\prime 2}\left|\psi_{0}\left(r^{\prime}\right)\right|^{2},
$$

where $\psi_{0}$ is the single-particle wave function for the halo with the normalization $4 \pi \int_{0}^{\infty} d r^{\prime} r^{\prime 2}\left|\psi_{0}\left(r^{\prime}\right)\right|^{2}=N=$ $M / m$, with $M$ being the total halo mass. Because in the SFDM model, all DM particles are in a single wave function, $\psi_{0}$ is enough for the current calculation. We expect the dominant contribution of the entanglement $S_{E}$ to be concentrated on the boundary region between region $\mathrm{A}$ and region B, as usual. However, in this paper, we do not separately consider the boundary effect.

Using $M_{A}(r)$, we can obtain the entanglement between $\mathrm{A}$ and $\mathrm{B}$ as a function of $r$ from Eq. (10). Then, the rotation velocity

$$
V_{\text {rot }}(r)=\sqrt{\frac{G M_{A}(r)}{r}},
$$

which can be observed, is directly related to the entanglement

$$
S_{E}(r) \simeq \frac{1}{2} \ln \left(\frac{r V_{r o t}^{2}(r)}{G M_{\odot}}\right)+102.42
$$

hence, we can infer the DM entanglement by observing the rotation curves of the galaxy.

To be concrete, let us consider a dwarf galaxy. Dwarf galaxies are the smallest DM-dominated objects and are ideal for DM study. The ground state of the SPEs is known to explain the rotation curves of dwarf galaxies well [22, 37-40]. The SPEs for a dwarf galaxy have an approximate analytic solution $\psi_{0}(r)=\psi_{0}(0) e^{-r^{2} / r_{c}^{2}} \quad$ 15] for the single-particle ground state, where $r_{c}$ is the core radius of the DM halo.

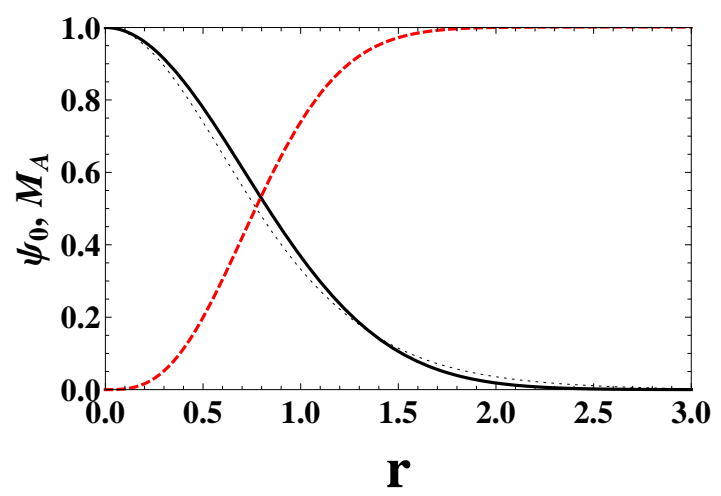

FIG. 1: (Color online) The approximate dark matter wave function $\psi_{0}$ (solid line) for a small galactic halo and the rescaled mass inside $r, M_{A}(r) / M$ (red dashed line), as functions of distance $r$ (in units of the halo core radius $r_{c}$ ) from the halo center. The dotted line represents the numerical solution of the SPE for $\psi_{0}$.

The wave functions are rescaled as $\psi_{0}(0)=1$.

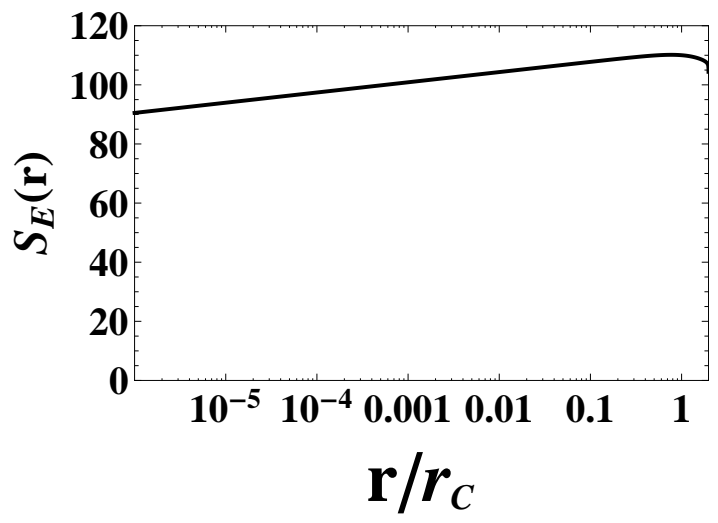

FIG. 2: Entanglement entropy between a central spherical region and the outside of the region as a function of $r$ up to $3 r_{c}$.

Figure 1 shows the above approximate solution, which looks quite similar to the numerical solution to the SPEs. The approximate solution has no sharp boundary, and an arbitrary a spatial cut off is chosen at $3 r_{c}$. Then, the normalization condition $4 \pi \int_{0}^{3 r_{c}} d r r^{2}\left|\psi_{0}(r)\right|^{2}=N$ gives $\psi_{0}(0) \simeq \alpha r_{c}^{-3 / 2} N^{1 / 2}$ with $\alpha=0.713$. Thus, the approxi- 


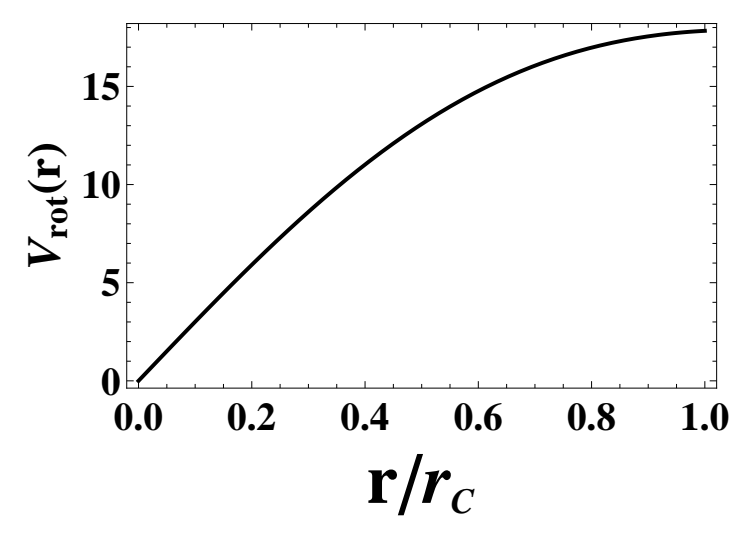

FIG. 3: Rotation velocity in $\mathrm{km} / \mathrm{s}$ of the model halo with $r_{c}=100 p c$.

mate ground-state wave function is

$$
\psi_{0}(r)=\alpha N^{1 / 2} \frac{e^{-r^{2} / r_{c}^{2}}}{r_{c}^{3 / 2}}
$$

and inserting Eq. (14) into Eq. (11) gives

$$
M_{A}(r)=\frac{\pi \alpha^{2}}{4}\left(\sqrt{2 \pi} \operatorname{erf}\left[\frac{\sqrt{2} r}{r_{c}}\right]-4 e^{-\frac{2 r^{2}}{r_{c}^{2}}} \frac{r}{r_{c}}\right) M
$$

where erf denotes the error function. Now inserting $M_{A}(r)$ into Eq. (10) gives $S_{E}(r)$ as shown in Fig. 2.

Figure 1 and 2 show an example with $M=10^{7} M_{\odot}$, which can be a model for a dwarf galaxy. For comparison, the wave function is rescaled, and $M(r)$ is given in units of $M$. Figure 2 shows $S_{E}(r)$, which is a rapidly rising function for small $r$ and approaches a maximum value as $M_{A}(r)$ approaches $M / 2$. One should notice that our approximate formula for $S_{E}$ is valid only for $N_{A}, N_{B} \gg 1$; hence, it is invalid as $r \rightarrow 0$ or as $r \rightarrow \infty$. However, obviously $S_{E}(r=0)=0$ because $|\Psi\rangle=|0\rangle_{A}|N\rangle_{B}$ if $N_{A}=0 . S_{E}$ is not small even for a small $r$ because the particle mass $m$ is extremely small compared to $M_{A}(r)$. Even a tiny region (say the solar system) has a gigantic number of DM particles that can be entangled with other regions. Due to the long wavelength of the BEC DM particles, all parts of a galaxy are entangled with other parts of the galaxy. Figure 3 shows the rotation curve for the model galaxy. Recall that $S_{E}(r)$ is related to $V_{r o t}(r)$ via Eq. (13).

\section{ENTANGLEMENT AND INTERFERENCE}

How can we detect the entanglement of DM in the sky? We can utilize the separability criterion based on interference for an atomic BEC in labs for the SFDM [52, 54]. Let us consider three cases: 1) typical CDM, 2) separable bosonic quantum DM with a fixed $N$, and 3 ) entangled bosonic quantum DM with a fixed $N$ like the SFDM. CDM particles such as WIMPs (weakly interacting massive particles) are usually treated as classical point particles without coherence. Therefore, we cannot expect any macroscopic quantum interference pattern or entanglement in galaxies made of typical CDM.

For separable DM states, one may expect some interference effects. However, the separable states cannot have perfect interference for a fixed $N$. This can be shown by following the arguments for a BEC in Refs. 52 and 54. All bipartite separable states have a density matrix in the form of

$$
\rho=\sum_{j} p_{j} \rho_{A}^{(j)} \otimes \rho_{B}^{(j)}
$$

where $\rho_{A}^{(j)}$ represents the $j$-th density matrix for the subsystem $A$. Then, according to the uncertainty principle, the separable states should satisfy the following inequality:

$$
\left((\Delta N)^{2}+1\right)\left(\left(\Delta\left(a_{A}-a_{B}\right)\right)^{2}+1\right) \geq \frac{\langle N\rangle_{\rho}}{4}+\frac{1}{8},
$$

where the real-valued variance for an observable $A$ is $(\Delta A)^{2} \equiv\left\langle A^{\dagger} A\right\rangle_{\rho}-\left|\langle A\rangle_{\rho}\right|^{2}$ for a given density matrix $\rho$ and $\langle N\rangle_{\rho}=N$ is the total number of DM particles. This inequality is from the Cauchy-Schwarz inequality with the number operator $N=a^{\dagger} a$. (See Eq. (4) of Ref. $54)$. Thus, the separable states cannot have a very small particle number variation $\Delta N$ and perfect interference (i.e., $\left.\left(\Delta\left(a_{A}-a_{B}\right)\right)^{2}=0\right)$ at the same time for large $N$. Separable states with $(\Delta N)^{2}>O(N)$ can have clear interference [54], but no quantum DM model in this class has been found so far. Thus, we will not consider this exotic case in this paper. (The total mass or $N$ of halo DM is related to the gravitational influence of DM on astronomical objects around the halos. In a sense, this gives a continuous measurement of $N$ by the objects; hence, we expect the DM state describing the halos as a whole to be one with almost fixed $N$.)

On the other hand, entangled states can have very small particle number variation and clear interference at the same time, violating the inequality in Eq. (17) 54]. Therefore, the presence of a clear interference pattern in the DM distribution can be a plausible criterion for DM entanglement. This interference pattern in the halo's DM density distribution might be inferred from future precise observations of rotation curves, gravitational lensing [55], and pulsa timing [56, 57], as well as from the data acquired by gravitational wave detectors [58].

As a toy example, we consider the DM interference fringes of two colliding galaxies $A$ and $B$ [59]. Note that now the regions $A$ and $B$ belong to two different colliding halos. If the SFDM particles in these galaxies are entangled, we would observe interferences violating the inequality in Eq. (17). This situation is quite similar to the interference experiment using an atomic BEC in a double-well potential in labs, so we can rely on the mathematics for an atomic BEC for DM. By precisely 


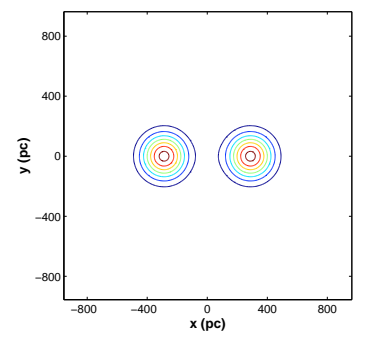

(a)

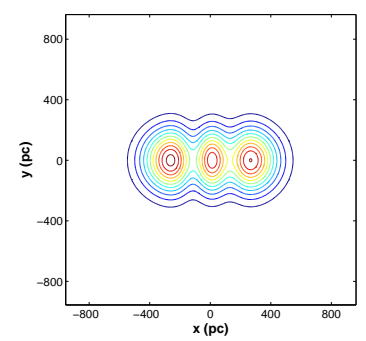

(b)

FIG. 4: A results of a numerical simulation showing the DM density distribution on the $x-y$ plane (a) before and (b) during the collision of two equal galaxies with total mass $M=10^{7} M_{\odot}$ and $\theta=0.2$. The interference

fringe is clearly shown in (b) at $t=0.8 \mathrm{Myr}$.

observing the rotation curves or gravitational lensing, one can deduce the DM density distribution and $\langle N\rangle_{\rho}$, which is proportional to the total mass $M=m\langle N\rangle_{\rho}$.

The density matrix $\rho=\left|\Psi_{0}\right\rangle\left\langle\Psi_{0}\right|$ for the state in Eq. (6) of the SFDM has a fixed $N$. Then, $\left(\Delta\left(a_{A}-a_{B}\right)\right)^{2}$, which is related to the variance of the phase difference between modes $A$ and $B$ (i.e., two colliding DM halos), becomes a crucial quantity for the separability test. If the DM particles in each mode maintain the same phase, this variance should be exactly zero, which is impossible for separable states with a fixed $N$. A high-precision numerical study with the SFDM [9] predicts the interference fringes made by the colliding DM halos. Therefore, we expect the quantum entanglement to be universal in SFDM-dominated cosmic structures.

In astronomical situations directly measuring the phase of the wave functions is difficult. However, because the variance $\left(\Delta\left(a_{A}-a_{B}\right)\right)^{2} \leq\left\langle\left(a_{A}^{\dagger}-a_{B}^{\dagger}\right)\left(a_{A}-a_{B}\right)\right\rangle_{\rho} \equiv$ $N_{b}$, as a check of the inequality, measuring

$$
\begin{aligned}
N_{b} & \equiv\left\langle b^{\dagger} b\right\rangle_{\rho}=\left\langle a_{A}^{\dagger} a_{A}+a_{B}^{\dagger} a_{B}-a_{A}^{\dagger} a_{B}-a_{B}^{\dagger} a_{A}\right\rangle_{\rho}(18) \\
& =N_{A}+N_{B}-\left\langle a_{A}^{\dagger} a_{B}+a_{B}^{\dagger} a_{A}\right\rangle_{\rho},
\end{aligned}
$$

which is the particle number in mode $b \equiv a_{A}-a_{B}$, is sufficient 54.

To understand the meaning of the last correlation term, we examine two colliding galactic halos with a relative phase $\theta$ and a $50: 50$ population ratio. The creation operators for this system are

$$
\begin{aligned}
& a_{A}^{\dagger}=\frac{1}{\sqrt{2}} \int d^{3} \mathbf{r} \psi_{A}(\mathbf{r}, t) \hat{\Psi}^{\dagger}(\mathbf{r}), \\
& a_{B}^{\dagger}=\frac{1}{\sqrt{2}} \int d^{3} \mathbf{r} \psi_{B}(\mathbf{r}, t) \hat{\Psi}^{\dagger}(\mathbf{r}),
\end{aligned}
$$

where $\psi_{A}(\mathbf{r}, t) \equiv u_{A}(\mathbf{r}, t) e^{i Q_{A} \mathbf{r}}$ is the time-dependent single-particle wave function of the halo $A$ with an initial wave vector $Q_{A}$; similarly, $\psi_{B}(\mathbf{r}, t) \equiv u_{B}(\mathbf{r}, t) e^{i Q_{B} \mathbf{r}}$ [60]. $Q_{A}$ and $Q_{B}$ of the galaxies can be estimated from the physical properties of visible matter, such as the colliding velocity of hydrogen gas in each galaxy. The $u_{A, B}$ is a real function with normalization $\int d^{3} \mathbf{r} u_{A, B}^{2}=1$. Then, the time-dependent entangled state similar to $\left|\Psi_{0}\right\rangle$ describing the pair of halos is

$$
|N, t\rangle \equiv \frac{1}{\sqrt{2^{N} N !}}\left(\int d^{3} \mathbf{r} \psi_{A}(\mathbf{r}, t) e^{i \theta / 2}+\psi_{B}(\mathbf{r}, t) e^{-i \theta / 2}\right)^{N} \hat{\Psi}^{\dagger}(\mathbf{r})|0\rangle,
$$

which is similar to the result for colliding two-BEC systems in the lab. This time-dependent many-body quantum state represents the two colliding BEC DM halos $(A$ and $B)$ with a relative global phase difference $\theta$ in their single-particle wave functions $\psi_{A}$ and $\psi_{B}$, respectably. Now, we consider the Fourier component of the DM density distribution with a relative wave vector $Q \equiv Q_{A}-Q_{B}$

$$
\begin{aligned}
\left\langle\rho_{Q}\right\rangle \equiv & \left\langle N, t\left|\int d^{3} \mathbf{r} \hat{\Psi}^{\dagger}(\mathbf{r}) \hat{\Psi}(\mathbf{r}) e^{i Q \mathbf{r}}\right| N, t\right\rangle \\
= & \langle N, t| \int d^{3} \mathbf{r}\left(a_{A}^{\dagger} u_{A} e^{-i Q_{A} \mathbf{r}}+a_{B}^{\dagger} u_{B} e^{-i Q_{B} \mathbf{r}}\right) \\
& \left(a_{A} u_{A} e^{i Q_{A} \mathbf{r}}+a_{B} u_{B} e^{i Q_{B} \mathbf{r}}\right) e^{i Q \mathbf{r}}|N, t\rangle \\
= & \left\langle N, t\left|a_{A}^{\dagger} a_{B}\right| N, t\right\rangle \\
= & \left\langle N-1, t\left|\frac{N e^{-i \theta}}{2}\right| N-1, t\right\rangle .
\end{aligned}
$$

One can obtain the second equality by inverting Eq. (19) and inserting $\hat{\Psi}(\mathbf{r})$. The third equality came from the condition $Q=Q_{A}-Q_{B}$. After some straightforward calculations, one can obtain the last equality. (See Eq. (29) of Ref. 60 for details.) We find $\left\langle\rho_{Q}^{\dagger}\right\rangle=\left\langle\rho_{-Q}\right\rangle=$ $\left\langle N, t\left|a_{B}^{\dagger} a_{A}\right| N, t\right\rangle=N e^{i \theta} / 2$. Therefore, for the entangled state $|N, t\rangle, N_{b}$ in Eq. (18) becomes

$$
N_{b}=N_{A}+N_{B}-\left\langle N, t\left|\rho_{Q}+\rho_{Q}^{\dagger}\right| N, t\right\rangle=N-N \cos (\theta),
$$

which violates the inequality (Eq. (17)) theoretically when $\theta<\cos ^{-1}(3 / 4)=0.723$ for large $N$. (This relation could also be useful for detecting the entanglement of an atomic BEC.) In a sense, $N_{b}$ measures a destructive interference. ( $N_{b}$ is maximum for $\theta=\pi$.)

How can we observe $N_{b}$ astronomically in this case? One might obtain $\left\langle\rho_{Q}\right\rangle$ and, hence $N_{b}$ by carefully observing the DM density distribution

$$
\rho(\mathbf{r}) \equiv\left\langle N, t\left|\hat{\Psi}^{\dagger}(\mathbf{r}) \hat{\Psi}(\mathbf{r})\right| N, t\right\rangle
$$


in the colliding galaxies by observing rotation curves or gravitational lensing and taking the Fourier transformation of $\rho(\mathbf{r})$ by using Eq. (21) with an estimated $Q$. Then, checking the deviation of the following inequality from Eq. (17)

$$
N_{b} \geq \frac{N}{4}-\frac{7}{8}
$$

is enough to decide the separability for the example we considered. In this way, the entanglement of galactic DM can be detected, in principle, by observing interferences, albeit technically challenging. Similarly, if we use $\left\langle\left(a_{A}^{\dagger}+a_{B}^{\dagger}\right)\left(a_{A}+a_{B}\right)\right\rangle_{\rho}=N+N \cos (\theta)$, we can detect a constructive interference. As a byproduct, one can obtain the relative phase difference $\theta$ of two halos, too.

Figure 4 shows an example of an interference pattern obtained from a three-dimensional numerical solution of the SPEs. We assumed Gaussian wave packets for the two initial halos with $\theta=0.2$ and an initial relative velocity $4 \times 10^{-4} c$. Then, we solved the SPEs by using the fast Fourier transformation code 61]. Separable DM such as CDM cannot have such a clear interference pattern as described above.

\section{DISCUSSION}

We show that, in the SFDM theory, two arbitrary regions of a galaxy are entangled even when no selfinteraction occurs among DM particles due to the nature of a BEC. The entanglement between a region and other parts of the current observable universe can be similarly estimated using a formula. A rough estimate gives $S_{E} \simeq \frac{1}{2} \ln \left(M_{u} / M_{\odot}\right)+102.42 \simeq 127.74$ at least, where
$M_{u}=O\left(10^{22}\right) M_{\odot}$ is the total DM mass of the universe. The inflation period in the early universe might have provided a chance for quantum coherence among widely separated cosmic structures; however, whether actual entanglement occurs among independent galactic halos is unclear. The entanglement of SFDM we consider is not from the inflationary phase, but from the generic property of a BEC. On the other hand, CDM particles such as WIMPs are usually very massive, and their number density is too low to have a condensation. Thus, we expect CDM particles not to have the macroscopic entanglement considered in this paper.

Because SFDM with an ultra-light mass can be a viable alternative to $\mathrm{CDM}$, understanding the observational differences between the two models is important. The SFDM can show a macroscopic quantum behavior because this DM has galactic-scale correlation and coherence, and it is robust against the decoherence induced by light or visible matter while conventional CDM particles are usually thought to be classical incoherent objects. A clear interference fringe in DM halos can appear in the SFDM model as a result of entanglement. Therefore, entanglement of DM can be another criterion that can be used to distinguish SFDM from CDM, for which observational effects, such as interference fringes, are worth of more studies.

\section{ACKNOWLEDGMENTS}

This work was supported by a Jungwon University Research Grant (2016-040).

\section{REFERENCES}

[1] W. H. Press, B. S. Ryden, and D. N. Spergel, Physical Review Letters 64, 1084 (1990).

[2] P. Salucci, F. Walter, and A. Borriello, Astronomy and Astrophysics 409, 53 (2003).

[3] J. F. Navarro, C. S. Frenk, and S. D. M. White, Astrophys. J. 462, 563 (1996).

[4] W. J. G. de Blok, A. Bosma, and S. S. McGaugh, astro-ph/0212102 (2002).

[5] A. Tasitsiomi, International Journal of Modern Physics D 12, 1157 (2003).

[6] S.-J. Sin, Phys. Rev. D50, 3650 (1994).

[7] J.-W. Lee and I.-G. Koh, Phys. Rev. D53, 2236 (1996).

[8] W. Hu, R. Barkana, and A. Gruzinov, Phys. Rev. Lett. 85, 1158 (2000).

[9] H.-Y. Schive, T. Chiueh, and T. Broadhurst, Nature Physics 10, 496 (2014).

[10] H.-Y. Schive et al., Phys. Rev. Lett. 113, 261302 (2014).

[11] J.-W. Lee, Journal of Korean Physical Society 54, 2622 (2009).
[12] A. Suárez, V. H. Robles, and T. Matos, Astrophysics and Space Science Proceedings 38, 107 (2014).

[13] T. Rindler-Daller and P. R. Shapiro, Modern Physics Letters A 29, 30002 (2014).

[14] T. Harko, Phys. Rev. D89, 084040 (2014).

[15] P.-H. Chavanis, Phys. Rev. D84, 043531 (2011).

[16] K. Huang, C. Xiong, and X. Zhao, International Journal of Modern Physics A 29, 50074 (2014).

[17] M. R. Baldeschi, G. B. Gelmini, and R. Ruffini, Physics Letters B 122, 221 (1983).

[18] M. Membrado, A. F. Pacheco, and J. Sañudo, Phys. Rev. A39, 4207 (1989).

[19] L. M. Widrow and N. Kaiser, Astrophys. J. Lett. 416, L71 (1993).

[20] F. E. Schunck, astro-ph/9802258 (1998).

[21] U. Nucamendi, M. Salgado, and D. Sudarsky, Phys. Rev. Lett. 84, 3037 (2000).

[22] A. Arbey, J. Lesgourgues, and P. Salati, Phys. Rev. D 64, 123528 (2001).

[23] A. Arbey, J. Lesgourgues, and P. Salati, Phys. Rev. D 65, 083514 (2002).

[24] J. Goodman, New Astronomy Reviews 5, 103 (2000). 
[25] P. Peebles, Astrophys. J. 534, L127 (2000).

[26] E. W. Mielke and F. E. Schunck, Phys. Rev. D 66, 023503 (2002).

[27] V. Sahni and L. Wang, Phys. Rev. D 62, 103517 (2000).

[28] M. Alcubierre et al., Class. Quant. Grav. 19, 5017 (2002).

[29] C.-G. Park, J.-c. Hwang, and H. Noh, Phys. Rev. D86, 083535 (2012).

[30] P. Sikivie and Q. Yang, Physical Review Letters 103, 111301 (2009).

[31] B. Fuchs and E. W. Mielke, Mon. Not. Roy. Astron. Soc. 350, 707 (2004).

[32] T. Matos, F. S. Guzman, L. A. Urena-Lopez, and D. Nunez, astro-ph/0102419 (2001).

[33] M. P. Silverman and R. L. Mallett, Classical and Quantum Gravity 18, L103 (2001).

[34] U. Nucamendi, M. Salgado, and D. Sudarsky, Phys. Rev. D 63, 125016 (2001).

[35] A. A. Julien Lesgourgues and P. Salati, New Astronomy Reviews 46, 791 (2002).

[36] C. G. Boehmer and T. Harko, JCAP 0706, 025 (2007).

[37] F. S. Guzman and T. Matos, Class. Quant. Grav. 17, L9 (2000).

[38] J. P. Mbelek, Astron. Astrophys. 424, 761 (2004).

[39] T. H. Lee and B. J. Lee, Phys. Rev. D 69, 127502 (2004).

[40] F. S. Guzman and F. D. Lora-Clavijo, Gen. Rel. Grav. 47, 21 (2015).

[41] L. Hui, J. P. Ostriker, S. Tremaine, and E. Witten, 1610.08297 (2016).

[42] J.-W. Lee, Phys. Lett. B681, 118 (2009).

[43] J.-W. Lee, Phys. Lett. B756, 166 (2016).

[44] H. L. Bray, arXiv1004.4016 (2010).

[45] F. S. Guzman, F. D. Lora-Clavijo, J. J. Gonzalez-Aviles, and F. J. Rivera-Paleo, JCAP 1309, 034 (2013).
[46] M. A. Nielsen and I. L. Chuang, Quantum Computation and Quantum Information (Cambridge University Press, Cambridge, 2001).

[47] J.-W. Lee, J. Lee, and H.-C. Kim, JCAP 0708, 005 (2007).

[48] J.-W. Lee, H.-C. Kim, and J. Lee, J. Korean Phys. Soc. 66, 1025 (2015).

[49] M. Van Raamsdonk, Gen. Rel. Grav. 42, 2323 (2010), [Int. J. Mod. Phys.D19,2429(2010)].

[50] E. Martin-Martinez and N. C. Menicucci, Class. Quant. Grav. 29, 224003 (2012).

[51] Y. Nambu, Phys. Rev. D78, 044023 (2008).

[52] C. Simon, Phys. Rev. A 66, 052323 (2002).

[53] W. Ding and K. Yang, Phys. Rev. A 80, 012329 (2009).

[54] G. Tóth, C. Simon, and J. I. Cirac, Phys. Rev. A 68, 062310 (2003).

[55] A. X. Gonzalez-Morales, A. Diez-Tejedor, L. A. UrenaLopez, and O. Valenzuela, Phys. Rev. D87, 021301 (2013).

[56] A. Khmelnitsky and V. Rubakov, JCAP 1402, 019 (2014).

[57] A. Aoki and J. Soda, Phys. Rev. D93, 083503 (2016).

[58] A. Aoki and J. Soda, arXiv:1608.05933 (2016).

[59] J. A. Gonzalez and F. S. Guzman, Phys. Rev. D83, 103513 (2011).

[60] A. Imambekov, V. Gritsev, and E. Demler, eprint arXiv:cond-mat/0703766 (2007).

[61] A. Paredes and H. Michinel, Phys. Dark Univ. 12, 50 (2016). 